\title{
Quantification of mutant SPOP proteins in prostate cancer using mass spectrometry-based targeted proteomics
}

\author{
Hui Wang ${ }^{1}$, Christopher E. Barbieri ${ }^{2}$, Jintang He${ }^{1}$, Yuqian Gao ${ }^{1}$, Tujin Shi' , Chaochao Wu', \\ Athena A. Schepmoes ${ }^{1}$, Thomas L. Fillmore', Sung-Suk Chae'², Dennis Huang'2, Juan Miguel Mosquera², \\ Wei-Jun Qian', Richard D. Smith', Sudhir Srivastava ${ }^{3}$, Jacob Kagan³, David G. Camp II', Karin D. Rodland ${ }^{1}$, \\ Mark A. Rubin² and Tao Liu' ${ }^{1 *}$
}

\begin{abstract}
Background: Speckle-type POZ protein (SPOP) is an E3 ubiquitin ligase adaptor protein that functions as a potential tumor suppressor, and SPOP mutations have been identified in 10\% of human prostate cancers. However, it remains unclear if mutant SPOP proteins can be utilized as biomarkers for early detection, diagnosis, prognosis or targeted therapy of prostate cancer. Moreover, the SPOP mutation sites are distributed in a relatively short region with multiple lysine residues, posing significant challenges for bottom-up proteomics analysis of the SPOP mutations.

Methods: To address this issue, PRISM (high-pressure, high-resolution separations coupled with intelligent selection and multiplexing)-SRM (selected reaction monitoring) mass spectrometry assays have been developed for quantifying wild-type SPOP protein and 11 prostate cancer-derived SPOP mutations.

Results: Despite inherent limitations due to amino acid sequence constraints, all the PRISM-SRM assays developed using Arg-C digestion showed a linear dynamic range of at least two orders of magnitude, with limits of quantification ranged from 0.1 to $1 \mathrm{fmol} / \mu \mathrm{g}$ of total protein in the cell lysate. Applying these SRM assays to analyze HEK293T cells with and without expression of the three most frequent SPOP mutations in prostate cancer (Y87N, F102C or F133V) led to confident detection of all three SPOP mutations in corresponding positive cell lines but not in the negative cell lines. Expression of the F133V mutation and wild-type SPOP was at much lower levels compared to that of F102C and Y87N mutations; however, at present, it is unknown if this also affects the biological activity of the SPOP protein.
\end{abstract}

Conclusions: In summary, PRISM-SRM enables multiplexed, isoform-specific detection of mutant SPOP proteins in cell lysates, providing significant potential in biomarker development for prostate cancer.

Keywords: Mass spectrometry, Targeted proteomics, SPOP mutation, Prostate cancer, Biomarker, PRISM-SRM

\section{Background}

Cancer is considered to be a genomic disease [1], and recent studies have identified mutations in speckle-type POZ protein (SPOP) in up to $15 \%$ of human primary prostate cancer $(\mathrm{PCa})$ patients $[2,3]$. SPOP mutations represent a distinct subclass of $\mathrm{PCa}$, in that tumors with

\footnotetext{
*Correspondence: tao.liu@pnnl.gov

1 Biological Sciences Division, Pacific Northwest National Laboratory, P.O. Box 999, MSIN: K8-98, Richland, WA 99354, USA

Full list of author information is available at the end of the article
}

SPOP mutations generally lack other common features of $\mathrm{PCa}$, including the PCa-specific TMPRSS2 and ETS family gene rearrangements (present in $\sim 50 \%$ of $\mathrm{PCa}$ ), abnormalities in the phosphatidylinositide 3-kinase pathway, or deletion and mutations of the tumor suppressor gene TP53 [2, 4-6]. Therefore, the presence of mutant SPOP may serve as a specific protein biomarker for early detection of prostate carcinogenesis, particularly in patients lacking the TMPRSS2-ETS gene fusions. The qualitative and quantitative study of mutant SPOP proteins can 
contribute to improved understanding of the functional significance and molecular mechanism of SPOP mutations in prostate cancer, and their relevance for targeted cancer therapies in clinical applications [7-11].

Currently available techniques for identification of SPOP mutation are mainly genomic methods, including whole-exome sequencing, Sanger sequencing, and quantitative RT-PCR [2, 12]. Although there have been several studies of the effects of forced over-expression of mutant SPOP protein in immortal cell lines [9-11], there appears to be no studies of endogenous SPOP mutations at the protein level in either primary prostate cancer cell lines or prostate cancer tissues, primarily due to the lack of antibodies capable of distinguishing between mutant and wild type SPOP protein. Traditional methods for protein measurement, which rely heavily on antibodies or other affinity reagents, are problematic for detection of SPOP mutations, for multiple reasons. First, most SPOP mutations in PCa are missense mutations, in which only one amino acid is altered, so specific antibodies against the mutant epitopes are required to distinguish the mutant isoforms from the wild type SPOP protein. This is a difficult task, even in such well-studied missense mutant proteins as TP53 and KRAS [13]. Also, various mutations can occur in the same amino acid position; for example, the most frequently mutated residue of SPOP, F133, displays four types of variants $(\mathrm{F} \rightarrow \mathrm{V} / \mathrm{L} / \mathrm{C} / \mathrm{S})$, making the antibody development much more difficult [2]. Moreover, seven amino acid (AA) residues covering the 11 most frequent mutations are clustered in a 49-AA region in SPOP, making it extremely difficult to develop mutationspecific antibodies in such an area. In this report we demonstrate that a highly sensitive targeted proteomic method, PRISM-SRM (high-pressure, high-resolution separations coupled with intelligent selection and multiplexing-selected reaction monitoring), is capable of providing quantitative information on both wild-type (WT) SPOP and four distinct single amino acid variants of SPOP that have been implicated in prostate cancer.

Mass spectrometry (MS)-based targeted proteomics methods such as SRM have become an increasingly important strategy for detection and quantitation of proteins in complex biological samples [14-18]. Combined with heavy isotope-labeled internal standards, targeted MS can provide highly sensitive and precise quantification of protein isoforms with a difference as small as a single AA residue. An example of this approach is the use of SRM-MS to identify and quantify multiple mutations of the Ras protein, either with enrichment by immunoprecipitation [13, 19] or SDS-PAGE [20]. Compared to Ras mutations where only two adjacent AA residues are involved, the most frequent SPOP mutations are distributed at seven distinct positions within a 49-AA region (AA 87-135) [2], requiring multiple distinct probes to fully characterize mutant SPOP proteins. Moreover, there are multiple lysine residues in this region, producing very small tryptic peptides including the mutated region that are unsuitable for bottom-up proteomics analysis. Herein, we describe the development of new SPOP mutation-specific SRM assays for highly sensitive and multiplexed quantification of multiple SPOP protein isoforms using combined Arg-C digestion and PRISM-SRM. The PRISM-SRM method developed at our laboratory provides both increased loading and reduced complexity, leading to higher sensitivity and specificity for low abundance protein quantification. It has been successfully applied to the quantitative analysis of several PCa-associated biomarkers, such as prostate-specific antigen (PSA) [21, 22], AGR2 [23] and TMPRSS2-ERG fusion proteins [5, 24]. The validity of these PRISM-SRM assays for quantifying low abundance SPOP proteins was demonstrated using several SPOP mutant cell lines, providing an effective method for verifying the mutant SPOP proteins as biomarkers in PCa.

\section{Methods \\ Materials}

Purified peptides ( $>97 \%$ purity) isotopically labeled with C-terminal $\left[{ }^{13} \mathrm{C}_{6}^{15} \mathrm{~N}_{2}\right]$ lysine or $\left[{ }^{13} \mathrm{C}_{6}^{15} \mathrm{~N}_{4}\right]$ arginine and their unlabeled counterparts were synthesized by Thermo Fisher Scientific (San Jose, CA). Urea, dithiothreitol (DTT), iodoacetamide (IAA) and formic acid (FA) were purchased from Sigma-Aldrich (St. Louis, $\mathrm{MO})$. Sequencing grade endoproteinases Arg-C and Asp- $\mathrm{N}$ were purchased from Roche (Indianapolis, IN). Recombinant SPOP protein was purchased from OriGene (Rockville, MD). The VCaP prostate cancer cells were obtained from the American Type Culture Collection (Manassas, VA).

\section{SPOP cell lines}

Wild-type (WT) and three SPOP mutants (Y87N, F102C and F133V) cell lines were established by transfecting human embryonic kidney (HEK) 293T cells with expression constructs encoding either WT SPOP or specific mutant SPOP sequences [2, 3].

\section{Protein extraction and digestion}

The cell line samples were lysed in $8 \mathrm{M}$ urea and $50 \mathrm{mM}$ $\mathrm{NH}_{4} \mathrm{HCO}_{3}$ with vortexing and sonication $(3 \times 20 \mathrm{~s})$, and the protein concentration was estimated using BCA assay (Pierce, Rockford, IL). Proteins were reduced with $10 \mathrm{mM} \mathrm{DTT}$ at $37^{\circ} \mathrm{C}$ for $1 \mathrm{~h}$, and subsequently alkylated with $40 \mathrm{mM}$ IAA at room temperature for $1 \mathrm{~h}$ in the dark. 
After tenfold dilution with $100 \mathrm{mM}$ Tris- $\mathrm{HCl}$ buffer $(\mathrm{pH}$ 7.6), $500 \mathrm{mM} \mathrm{CaCl}_{2}$ was added to the sample to reach a final concentration of $1 \mathrm{mM}$. Protein digestion was performed at an enzyme-to-protein ratio of $1: 50$, at $37^{\circ} \mathrm{C}$ for $3 \mathrm{~h}$. The enzymatic reaction was stopped by adding trifluoroacetic acid (TFA) solution to a final concentration of $0.1 \%$. The resulting peptide digests were then cleaned up by C18 SPE cartridge (Supelco, Bellefonte, PA). The recombinant SPOP protein sample was digested in the same way without the lysis step.

\section{LC-MS/MS analysis}

LC-MS/MS analyses of the Arg-C and Asp-N digests of recombinant SPOP protein were carried out on a nanoACQUITY UPLC (Waters, Milford, MA) coupled to an Orbitrap Velos mass spectrometer (Thermo Scientific). The LC separation was performed on a C18 column with $0.1 \%$ FA in $\mathrm{H}_{2} \mathrm{O} / \mathrm{ACN}$ as buffer $\mathrm{A} / \mathrm{B}$ at a gradient of $1-8 \% \mathrm{~B}$ in $0-2 \mathrm{~min}, 8-12 \% \mathrm{~B}$ in $2-20 \mathrm{~min}$, $12-30 \% \mathrm{~B}$ in $20-75 \mathrm{~min}, 30-45 \%$ in $75-97 \mathrm{~min}$, and $45-95 \%$ B in $97-100 \mathrm{~min}$. The heated capillary temperature and spray voltage was $350{ }^{\circ} \mathrm{C}$ and $2.2 \mathrm{kV}$, respectively. Full MS spectra were recorded at a resolution of 60,000 over the range of $\mathrm{m} / z 300-2000$. Ten parent ions with most abundant intensity were selected for MS/MS using CID with a collision energy of 35V. The resulting raw data were searched against the UniProt human protein database [25] using the MSGF+ algorithm [26, 27]. The parameter was $20 \mathrm{ppm}$ tolerance for precursor ion masses and 0.5 Da tolerance for fragment ions, with static carbamidomethylation on cysteine residues $(+57.0215 \mathrm{Da})$ and dynamic oxidation of methionine residues $(+15.9449 \mathrm{Da})$. The data filtering criteria is MSGF $\mathrm{E}<10^{-8}, \mathrm{Q}<0.01$ and mass measurement error $<20 \mathrm{ppm}( \pm 10 \mathrm{ppm})$.

\section{PRISM fractionation}

The PRISM pre-fractionation method has been described previously [21]. Briefly, peptide fractionation was performed on an in-house C18 $(3 \mu \mathrm{m}, 300 \AA$, Jupiter, Phenomenex, Torrance, CA) packed capillary column $(200 \mu \mathrm{m}$ i.d. $\times 40 \mathrm{~cm})$ at a flow rate $2.2 \mu \mathrm{L} /$ min on a Waters nanoACQUITY UPLC system. Buffers for high and low $\mathrm{pH}$ reversed-phase (RP) fractionation were $10 \mathrm{mM}$ of ammonium formate $\left(\mathrm{pH}\right.$ 9) in $\mathrm{H}_{2} \mathrm{O} / 90 \%$ $\mathrm{ACN}(\mathrm{A} / \mathrm{B}), 0.1 \%$ FA in $\mathrm{H}_{2} \mathrm{O} / \mathrm{ACN}(\mathrm{A} / \mathrm{B})$ and $0.1 \%$ TFA in $\mathrm{H}_{2} \mathrm{O} / \mathrm{ACN}(\mathrm{A} / \mathrm{B})$, respectively. The LC gradient was $0.5 \% \mathrm{~B}$ in $0-35 \mathrm{~min}, 0.5-20 \% \mathrm{~B}$ in $35-37 \mathrm{~min}, 20-55 \%$ $B$ in $37-107$ min and $55-90 \%$ B in $132-135$ min. Fortyfive micrograms of protein digests were injected for each PRISM run. The 1-min fractions were collected in vials of a 96-well plate and diluted to $20 \mu \mathrm{L}$ with water for the next dimensional LC-SRM analysis.

\section{SRM assay development}

The SRM transitions of each target peptide were optimized separately in direct infusion mode on a TSQ Vantage triple quadrupole mass spectrometer (Thermo Fisher Scientific). The predominant charge states of a peptide were selected as parent ions. Then, collision energy (CE) was ramped for each charged precursor to get the most intense fragments. Optimal CE value was determined by calculating the average value of three $\mathrm{CE}$ measurements. The transitions and optimal CE values were further validated by LC-SRM analysis of the heavy peptide standards spiked in a VCaP digest. Transition with low MS signal and high interference level were removed.

The method for determination of the calibration curve and the definition of the limit of detection (LOD) and limit of quantification (LOQ) has been described previously [5], with the addition of Arg-C digests from $\mathrm{VCaP}$ cell line at a final concentration of $1 \mu \mathrm{g} / \mu \mathrm{L}$ as a matrix, as described below. The reverse response curve for each SPOP peptide was generated by varying the amount of heavy peptide with a constant amount of light peptide ( $5 \mathrm{fmol} / \mu \mathrm{L}$ for the peptide in the non-mutant region, $10 \mathrm{fmol} / \mu \mathrm{L}$ for peptides covering potential mutant site F102 or F133 and $20 \mathrm{fmol} / \mu \mathrm{L}$ for a peptide including possible mutant site Y87). The twelve-point response curves $(0.05,0.1,0.25,0.5,1,2.5,5,10,25,50,100$ and $250 \mathrm{fmol} /$ $\mu \mathrm{L}$ for heavy peptides in non-mutant region, and twofold and fourfold of each concentration data point for heavy peptides containing mutation site F102/F133 and Y87, respectively) covered more than 2 orders of magnitude. Three replicates of each data point were analyzed.

\section{LC-SRM analysis}

All LC-SRM experiments were performed on a nanoACQUITY UPLC coupled to TSQ Vantage MS instrument, with $4.5 \mu \mathrm{L}$ of sample injection for each measurement. $0.1 \% \mathrm{FA}$ in water and $0.1 \%$ in $90 \% \mathrm{ACN}$ were used as buffer $\mathrm{A}$ and $\mathrm{B}$, respectively. Peptide separations were performed by an in-house C18 $(3 \mu \mathrm{m}, 300 \AA$, Jupiter) packed capillary column $(75 \mu \mathrm{m}$ i.d. $\times 40 \mathrm{~cm})$ at a flow rate of $350 \mathrm{~nL} / \mathrm{min}$ using gradient of $0.5 \% \mathrm{~B}$ in 0-14.5 min, $0.5-15 \%$ B in $14.5-15.0 \mathrm{~min}, 15-40 \% \mathrm{~B}$ in 15-30 min and $45-90 \%$ B in 30-32 min. The heated capillary temperature and spray voltage was set at $350{ }^{\circ} \mathrm{C}$ and $2.4 \mathrm{kV}$, respectively. Both the Q1 and Q3 were set as 0.7 FWHM. A scan width of $0.002 \mathrm{~m} / z$ and a dwell time of $10 \mathrm{~ms}$ were used. All the SRM data were analyzed by Skyline Software [28].

\section{Results \\ Peptide selection}

In contrast to wild-type proteins, peptide selection for mutant proteins is constrained to those peptides that 
cover the mutation sites. Consequently, some candidate peptides may not meet the "gold standard", e.g., 6-20 AA in length, good LC elution, and strong MS signal, which is typically considered for selection of target peptides. The most frequent SPOP mutations in prostate cancer are located at seven distinct AA residues (e.g., Y87, F102, W131 and F133) clustered in the MATH domain (AA 31-166) [2, 29]. The AA sequence of SPOP has eight lysine $(K)$ and three arginine $(R)$ residues between the first (Y87) and last mutation site (K134) in the MATH domain (shown in Fig. 1). Use of trypsin for digestion of SPOP is therefore impractical for LC-SRM analysis as most of the resulting tryptic peptides that include the mutation sites are too short. Short peptides often have weak retention on commonly used C18 RPLC column, and the choice of transitions for short peptides is much more limited (i.e., lower specificity). Additional file 1: Table S1 shows the peptide candidates covering the seven potential SPOP mutation sites generated by in silico digestion. Six of seven tryptic peptides have peptide length of less than 6 AA (Additional file 1: Table S1). Lys-C digestion has similar limitations due to a large number of lysine residues in this region.

Arg- $\mathrm{C}$, on the other hand, specifically hydrolyzes proteins and peptide bonds at the $\mathrm{C}$-terminal side of arginine residues; unlike trypsin, Arg- $\mathrm{C}$ does not cleave at the $\mathrm{C}$-terminal of lysine residues. The result of in silico digestion indicates that the Arg-C peptides can cover the majority of the SPOP mutation sites (Additional file 1: Table S1). Besides Arg-C, Asp-N which cleaves peptide bonds at the $\mathrm{N}$-terminal of aspartic acid (D) or glutamic acid (E) residues was also considered. All the Asp-N peptides covering the mutation sites are appropriate in length for SRM measurement (Additional file 1: Table
S1). To determine which enzymatic digestion generates more MS-responsive peptides that cover the mutation sites, these two proteases were used to digest recombinant SPOP protein, followed by LC-MS/MS analysis of the digests. Additional file 2: Figure S1 shows a heat map of the spectral counts of SPOP peptides generated by the two enzymatic digestions. For both conditions, the identification of peptides in the mutation region was more difficult compared to the non-mutation areas. The sequence coverage and relative peptide abundance by Arg- $\mathrm{C}$ in the entire protein, especially in the mutation region (Fig. 1), is much higher than that of Asp- $\mathrm{N}$ digestion; Arg-C also produced a total of 162 unique peptides, compared to 86 by Asp- $\mathrm{N}$, demonstrating the superior performance of Arg-C digestion for producing surrogate peptides for the SPOP protein isoforms. Therefore, Arg-C was selected for SPOP proteolysis, and PRISMSRM assays for a total of 16 SPOP peptide candidates were developed, including 11 mutation sites $(\mathrm{Y} 87 \mathrm{C} / \mathrm{N}$, F102C, K129C, D130H, W131C/G, F133L/V/S and $\mathrm{K} 134 \mathrm{~N}$ ) [2] and their related WT counterparts, as well as two "normal" peptides shared by all protein isoforms in the non-mutation region.

\section{Transition selection and validation}

Typically, the most intensive and interference-free precursor and fragment ions are selected as the transitions for the target peptides. However, transition selection for mutant proteins can be challenging, due to the high similarity in AA sequence between the point mutation peptides and its normal form, such that they generate similar series of fragment ions, especially if the mutation site is near the $\mathrm{C}$ - or $\mathrm{N}$-terminus. In this situation, distinguishing between mutant and normal peptides relies on

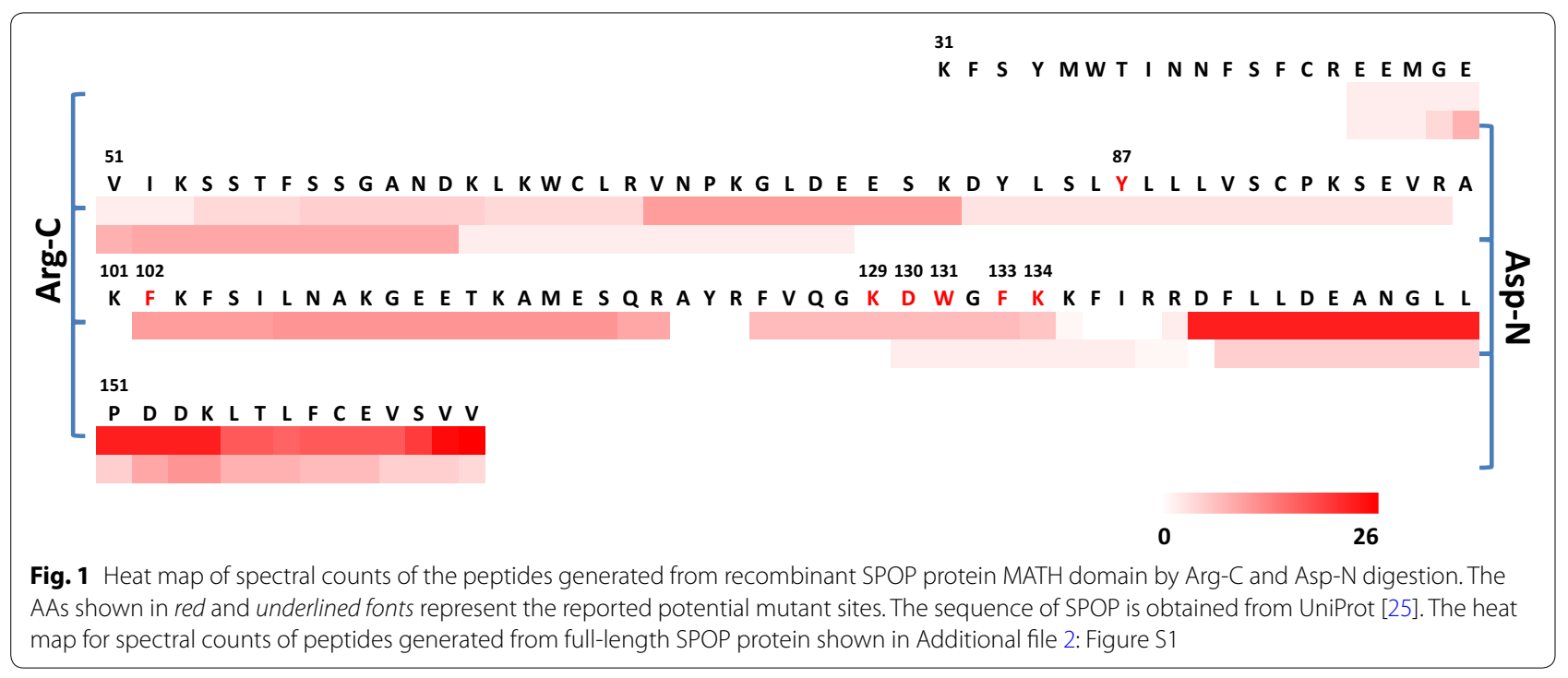


the precursor $m / z$; if their precursor $m / z$ values are also close, then additional information, such as the difference in LC elution time, is also needed.

Most of the Arg-C peptides covering the SPOP mutation site(s) have multiple internal lysine residues, and hence carry multiple charges with the $4+$ precursor ions being the most intense (Table 1). Compared to the common doubly charged precursors, the $\mathrm{m} / \mathrm{z}$ differences between mutant and normal precursors with $4+$ charges are much smaller. For instance, the mutant peptide VNPKGLDEESKDYLSLCLLLVSCPKSEVR and its WT peptide VNPKGLDEESKDYLSLYLLLVSCPKSEVR only have a small $\mathrm{m} / \mathrm{z}$ difference of 0.7581 , and share many intensive fragment ions, which is insufficient to distinguish them solely by the SRM transitions. In our LC-SRM analysis of the peptide mixture, we found two peaks appeared in the extracted ion chromatogram (XIC) of peptide VNPKGLDEESKDYLSLYLLLVSCPKSEVR (Peaks 1 and 2 in Additional file 3: Figure S2A), which was caused by coscanning of the WT peptide and its mutant counterpart VNPKGLDEESKDYLSLCLLLVSCPKSEVR. On the other hand, only Peak 1 was detected in the XIC of VNPKGLDEESKDYLSLCLLLVSCPKSEVR (Additional file 3: Figure S2B), suggesting that Peak 1 belongs to this mutant peptide while Peak 2 belongs to the WT peptide VNPKGLDEESKDYLSLYLLLVSCPKSEVR. The final validated, optimal transitions for the 16 target peptides are listed in Additional file 4: Table S2.

\section{Matrix effects on peptide loss}

During the transition validation, we also noted that some hydrophobic SPOP peptides showed a poor response in the LC-SRM analysis of the neat peptide mixture. One of the examples is that peptide VNPKGLD EESKDYLSLYLLLVSCPKSEVR and its two mutant forms VNPKGLDEESKDYLSLCLLLVSCPKSEVR and VNPKGLDEESKDYLSLNLLLVSCPKSEVR were barely detected in the neat peptide mixture (Additional file 5: Figure S3A). We postulated that the lack of a protective matrix in the pure peptide stocks might contribute to substantial loss of the highly hydrophobic peptides. To test this hypothesis, we prepared the heavy peptide stock solutions in a $\mathrm{VCaP}$ cell digest with a final concentration of $0.2 \mu \mathrm{g} / \mu \mathrm{L}$ and repeated the analysis. Indeed, all three heavy peptides from the stock solution with matrix were detected with much better response (Additional file 5: Figure S3B).

The matrix effect on all the 16 target peptides was further investigated by plotting the peptide recovery (peak area ratio of no-matrix versus with-matrix) to their theoretical hydrophobicity calculated based on AA sequence using the Thermo Fisher on-line peptide analyzing tool (http://www.thermofisher.com; Additional file 6: Figure S4). Overall, three of the peptides with hydrophobicity $<30$ showed high recovery $(>70 \%)$; six peptides with hydrophobicity $>32.5$ (color zone) have $\sim 10 \%$ recovery; three peptides with hydrophobicity $>40$ (the examples in Additional file 3: Figure S2) have only $2 \%$ recovery. This result demonstrated that adding a matrix with a similar peptide concentration level for LC-SRM analysis $(0.2 \mu \mathrm{g} /$ $\mu \mathrm{L})$ to the pure peptide stocks is an effective way to reduce peptide loss, especially for the hydrophobic peptides. Moreover, it also helped to reduce run-to-run variation, e.g., the $\mathrm{CV}$ of $\mathrm{H} / \mathrm{L}$ ratio of peptides reduced from 4 to $34 \%$ using no-matrix stocks (mean CV $\sim 14 \%$ ) to $<10 \%$ using the stocks with matrix (mean CV $\sim 4 \%$ ), demonstrating the importance of matrix on the stock stability of these Arg-C SPOP peptides. Two other matrices, tryptic digests of LNCaP prostate cancer cells and Shewanella oneidensis, were also tested and they were equally effective in reducing the loss of hydrophobic peptides and improving the reproducibility of LC-SRM analysis (data not shown).

\section{Peptide loss in PRISM fractionation}

Regular LC-SRM could not detect many of the low abundance SPOP proteins in the cells (see Additional file 7: Figure S5). Therefore, PRISM-SRM, a highly sensitive

Table 1 LOD and LOQ (fmol/ $\mu \mathrm{g}$ of total protein) of the PRISM-SRM assays for WT and mutant SPOP proteins

\begin{tabular}{|c|c|c|c|c|c|c|}
\hline Peptide & Charge & Transition & Production type & LOD fmol/ $/ \mathrm{gg}$ & $\mathrm{LOQ} \mathrm{fmol} / \mu \mathrm{g}$ & $t_{R} \min$ \\
\hline VNPKGLDEESKDYLSLYLLLVSCPKSEVR & $4+$ & $838.7-962.2$ & y8 & 0.4 & 1 & 30.0 \\
\hline VNPKGLDEESKDYLSLNLLLLVSCPKSEVR & $4+$ & $826.4-962.5$ & y8 & 0.2 & 0.4 & 28.4 \\
\hline AKFKFSILNAKGEETKAMESQR & $4+$ & $629.1-721.3$ & y6 & 0.5 & 1 & 24.5 \\
\hline AKCKKFSILNAKGEETKAMESQR & $4+$ & $632.3-721.3$ & y6 & 0.5 & 1 & 23.8 \\
\hline FVQGKDWGFKKFIR & $4+$ & $439.8-895.6$ & $y 7$ & 0.2 & 0.5 & 25.6 \\
\hline FVQGKDWGVKKKFIR & $4+$ & $427.8-847.6$ & y7 & 0.1 & 0.2 & 24.5 \\
\hline LADELGGLWENSR & $2+$ & 730.4-918.4 & y8 & 0.05 & 0.1 & 27.9 \\
\hline SLASAQCPFLGPPR & $2+$ & $750.9-943.5$ & y8 & 0.05 & 0.1 & 26.1 \\
\hline
\end{tabular}

$t_{R}$ retention time 
targeted quantification technique which has enabled quantification of low-abundance proteins at $\mathrm{pg} / \mathrm{mL}$ level in depleted plasma/serum or low $100 \mathrm{~s}$ copies per cell levels [30], was used for developing the SPOP mutation assays. Compared to regular LC-SRM, PRISMSRM allows for up to 50 times more loading (maximum $50 \mu \mathrm{g})$ of samples in the first dimension high $\mathrm{pH}(\mathrm{pH} 9)$ reversed-phase LC separation (with optional concatenation for multiplexing), specific target peptide-containing fractions with much-reduced sample complexity are then analyzed by LC-SRM, leading to as high as 100 times improvement in sensitivity [21].

However, not all peptides can take full benefit of the PRISM separation (e.g., due to potential peptide loss in the additional dimension of separation). We evaluated the recovery of the peptides after fractionation by comparing the heavy peptide intensity of PRISM-SRM to that of straight LC-SRM analysis. It was noted that all the peptides in the mutant region had a PRISM-SRM/LC-SRM ratio of $\leq 1$. Three peptides with significant sample loss have $\mathrm{p} I$ of $\sim 10$ or higher (Additional file 8: Figure S6). We reason that the sample loss may be caused by the strong interaction between the positively charged basic peptides and the negatively charged silanol groups on the separation media in the high $\mathrm{pH}$ separation condition $(\mathrm{pH}$ 9). To determine if acidic RP conditions can reduce the PRISM peptide loss, we tested $0.1 \%$ FA and $0.1 \%$ TFA in $\mathrm{H}_{2} \mathrm{O} / \mathrm{ACN}$ as the mobile phases A/B for the first-dimension PRISM fractionation. Indeed, the results showed that FA mobile phases slightly improved the overall peptide detection, while TFA mobile phases provided a significant boost of peptide signals, in particular for the peptides having high $\mathrm{p} I$ values (Additional file 8: Figure S6). Thus, $0.1 \%$ TFA mobile phases were selected for the optimal condition of SPOP PRISM fractionation. Overall, PRISM-SRM of these long, multiple-internal-lysine-containing Arg-C peptides did not provide even higher (e.g., $>100 \times$ ) improvement in sensitivity like the case of other "regular" peptides, presumably due to the reduced matrix protection in the PRISM fractions (i.e., the majority of the non-target peptides are removed) and the reduced orthogonality with the second-dimension acid RPLC separation. However, it still enabled much needed more sensitive measurements of the SPOP mutation peptides comparing to regular straight LC-SRM analysis.

\section{PRISM-SRM quantification}

To quantify the mutant SPOP peptides, the response curve, LOD and LOQ for eight SPOP peptides (three peptides represented as both WT and mutant versions, plus the two "common" peptides in the non-mutated region) in PRISM-SRM assay was determined. The result was shown in Fig. 2 and Table 1. Although the Arg-C digested SPOP peptides in the mutation region are longer and more hydrophobic, the mutation PRISM-SRM assays still showed an excellent linear dynamic range of more than 2 orders of magnitude (Fig. 2). The LOD and LOQ for SPOP peptides range from 0.05 to 0.5 and 0.1 to 1.0 $\mathrm{fmol} / \mu \mathrm{g}$, respectively. The median $\mathrm{CV}$ for each peptide in triplicate PRISM-SRM runs was $\leq 10 \%$, demonstrating good reproducibility. Furthermore, among all the mutant SPOP peptides, FVQGKDWGVKKFIR derived from mutant $\mathrm{SPOP}^{\mathrm{F} 133 \mathrm{~V}}$ has the best sensitivity for quantification by PRISM-SRM with the lowest LOD and LOQ values (Table 1). Interestingly, the $\mathrm{SPOP}^{\mathrm{F} 133 \mathrm{~V}}$ mutation is the most frequently mutated residue in prostate cancer ( $50 \%$ of the total SPOP mutations) [5]. Therefore, our newly developed point-mutation-specific PRISM-SRM assays could be very useful to study the most commonly observed SPOP protein mutations in prostate cancer.

The optimized PRISM-SRM assay was applied to quantitatively analyze protein digests from SPOP cell line samples. A total of 5 cell lines including HEK293T cells and HEK293T cells expressing either SPOP-WT or SPOPmutant (Y87N, F102C, and F133V) were tested. The XICs of SPOP peptides in HEK293T cell lines expressing SPOP-WT and three SPOP mutations by PRISM-SRM analysis are shown in Fig. 3 and Additional file 9: Figure S7. All the endogenous peptides, both WT and mutant peptides, were detected clearly in the corresponding transfected cell line samples, but not in the non-transfected HEK293T control sample (data not shown). Compared to the LC-SRM analysis, the increased loading and reduced background interference in PRISM-SRM enabled the detection of peptides in even the cell lines with lower SPOP protein expression, such as VNPK GLDEESKDYLSLYLLLVSCPKSEVR and AKFKFSIL NAKGEETKAMESQR in WT and F133V cells. The CV of peptide VNPKGLDEESKDYLSLNLLLVSCPKSEVR and AKCKFSILNAKGEETKAMESQR was calculated as 3.7 and $4.6 \%$, respectively. The CV for the mutant peptide FVQGKDWGVKKFIR's was $16.7 \%$ due to the lower abundance of the endogenous protein. Overall, the CV of each SPOP peptide in all samples was $\leq 20 \%$ showing good reproducibility of the PRISM-SRM assay.

The SPOP protein concentrations were calculated based on the peak area ratio and the response curve (Table 2). Although detected, peptide AKFKFSILNAKGEETKAMES QR in the SPOP-WT and F133V cell lines was below LOQ and could not be quantified. From the two peptides in the non-mutant region (LADELGGLWENSR and SLASAQCPF LGPPR), it can be seen that the overall expression of WT and F133V is 10-20 times lower than that of Y87N and F102C in the corresponding cell lines. The mutant peptides VNPK GLDEESKDYLSLNLLLVSCPKSEVR, AKCKKFSILNAKGE ETKAMESQR and FVQGKDWGVKKF in the three 

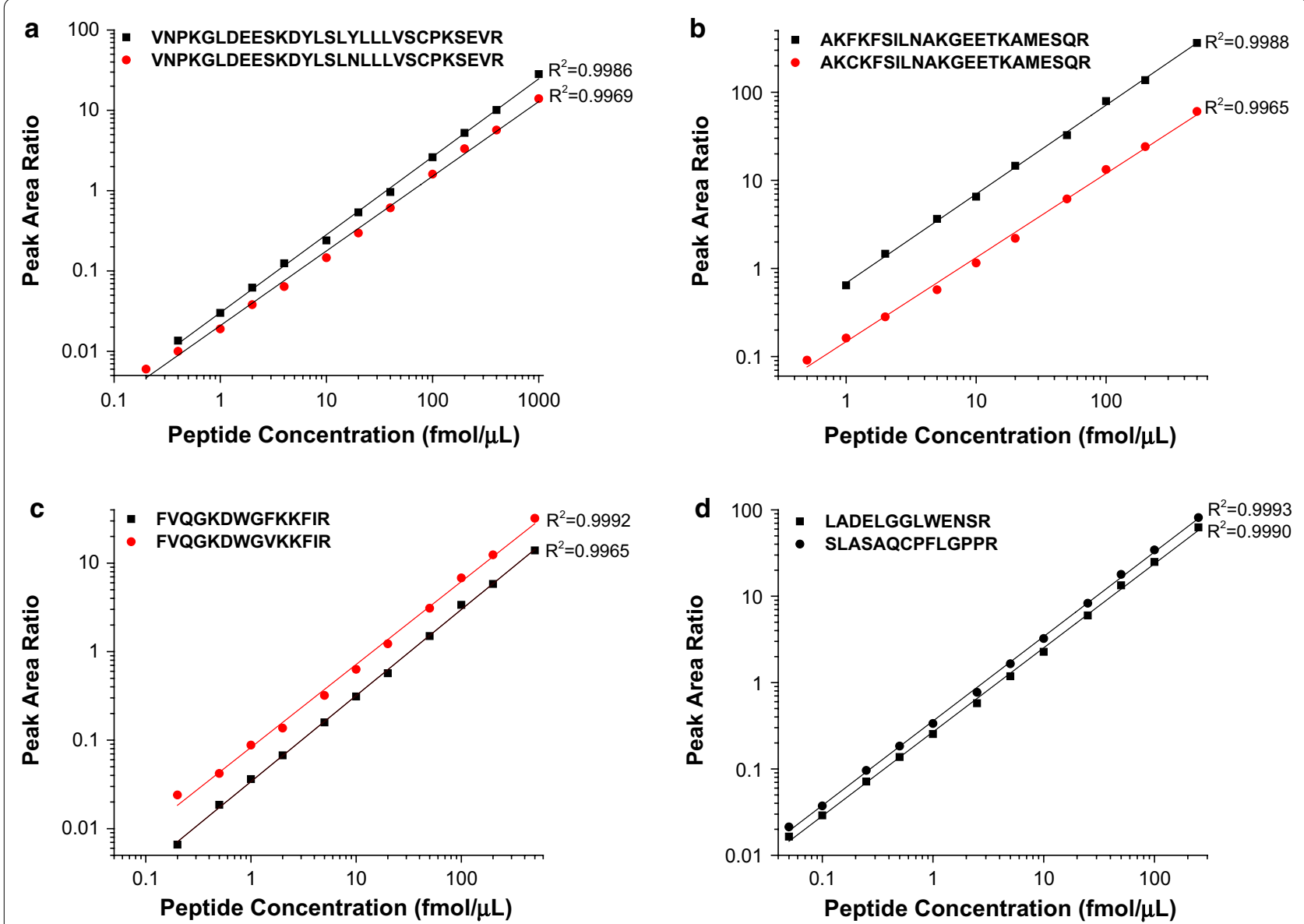

Fig. 2 The response curve of the PRISM-SRM assays (in log-log scale) for eight SPOP peptides (blackWT; red mutant): VNPKGLDEESKDYLSLYLLLVSC PKSEVR/VNPKGLDEESKDYLSLNLLLVSCPKSEVR (a), AKFKFSILNAKGEETKAMESQR/AKCKKFSILNAKGEETKAMESQR (b), FVQGKDWGFKKFIR/FVQGKDWGV $\operatorname{KKFIR~(c)~and~LADELGGLWENSR/SLASAQCPFLGPPR~(d)~}$

transfected cell lines were determined to be $61.4,9.4$ and $1.8 \mathrm{fmol} / \mu \mathrm{g}$ of total protein, respectively.

\section{Discussion}

Studies on protein mutations have relied heavily on isoform-specific antibodies. However, it is very challenging to develop high-quality antibodies for specific recognition of protein isoforms without cross-reactivity. Compared to antibody-based methods, MS-based targeted proteomics has higher specificity, shorter lead times and much higher success rates in developing sensitive, specific and accurate assays for protein isoform quantification. The high quality heavy isotope-labeled peptide standards needed for highly confident and precise quantification can also be obtained readily from commercial vendors. Moreover, front-end peptide enrichment methods such as SISCAPA (stable isotope standards and capture by anti-peptide antibodies) [31] and PRISM enable protein quantification at low $\mathrm{ng} / \mathrm{mL}$ to $50 \mathrm{pg} / \mathrm{mL}$ levels in blood plasma/serum, rivaling the sensitivity of ELISA assays. Thus, targeted proteomics provides a valuable alternative for preclinical validation of protein isoforms (e.g., mutation, splice variant, gene fusion, and post-translational modification) as potential disease biomarkers.

The SRM quantification of mutant SPOP proteins has some unique challenges that are inherently associated with its AA sequence: there are seven potential mutation sites distributed in a 49-AA region containing eight lysine and three arginine residues. This precludes the conventional enzymatic digestion methods using trypsin or Lys- $\mathrm{C}$ because most of the enzymatically cleaved peptides covering the mutation site(s) are too short to be analyzed by LC-MS/MS with good specificity. Arg-C produces longer peptides that cover all the mutation sites (14-29 AA). However, these peptides are in general less responsive in electrospray ionization, compared to typical tryptic peptides that are selected for SRM analysis (e.g., 6-20 AA). The presence of multiple internal lysine residues also causes the most MS-responsive precursor 

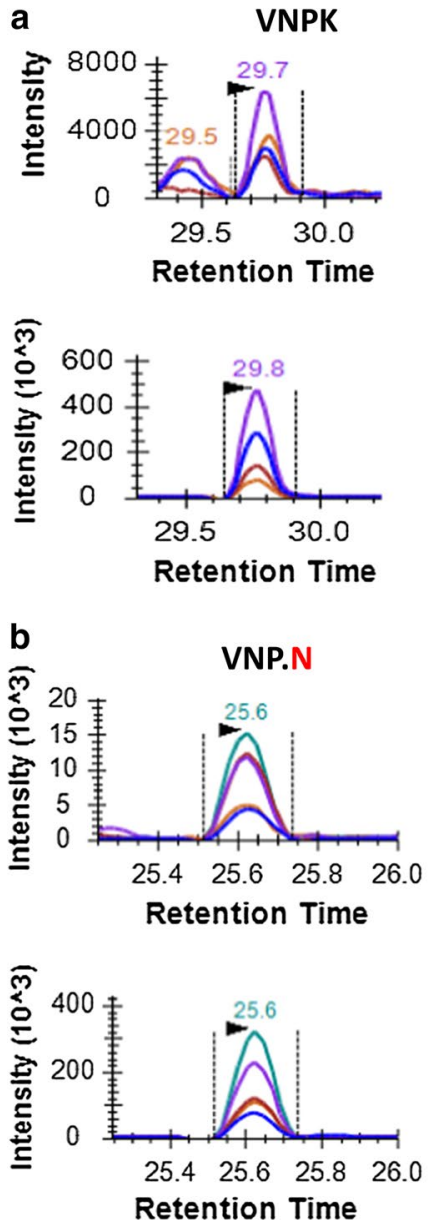
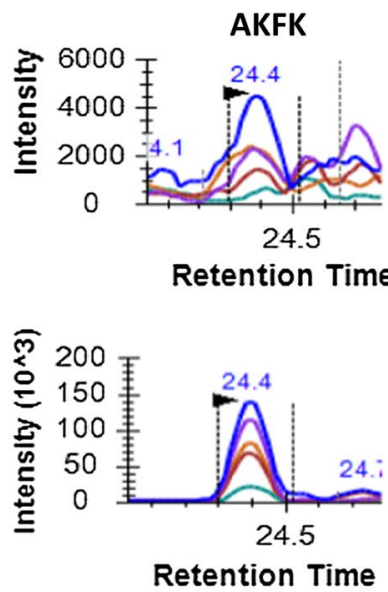

АKCK
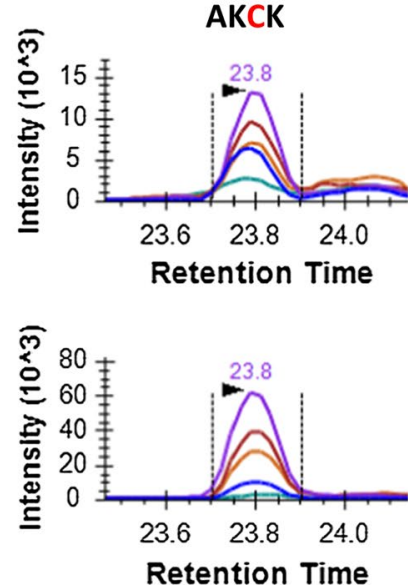
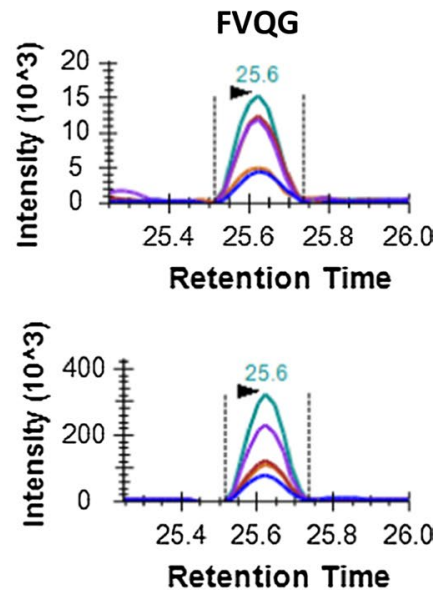

FVQ.V
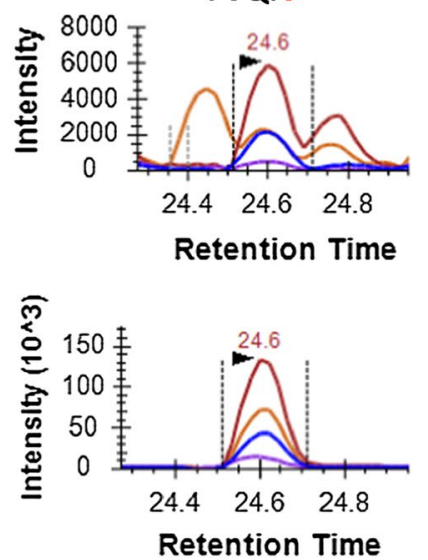

Fig. 3 XICS of SPOP WT peptides in mutant region detected in HEK293T cell lines expressing WT SPOP cell line (a) and their corresponding mutant counterparts detected in SPOP Y87N, F102C and F133V mutant cell lines (b) using PRISM-SRM (AA shown in red font indicates mutation site). XICS for all the peptide candidates (details available in Additional file 4: Table S2) in four SPOP cell lines were shown in Additional file 9: Figure S7

Table 2 Quantification of SPOP peptides in HEK293T cells expressing WT and mutant SPOP proteins by PRISM-SRM

\begin{tabular}{|c|c|c|c|c|c|c|c|c|}
\hline \multirow[t]{2}{*}{ Cell line } & \multicolumn{2}{|c|}{$\begin{array}{l}\text { VNPKGLDEESKDYLSLYYLLL- } \\
\text { VSCPKSEVR }\end{array}$} & \multicolumn{2}{|c|}{$\begin{array}{l}\text { AKFKFSILNAKGEET- } \\
\text { KAMESQR }\end{array}$} & \multicolumn{2}{|c|}{ FVQGKDWGFKKFIR } & \multirow{2}{*}{$\begin{array}{l}\text { LADELGGLWENSR } \\
\text { WT }\end{array}$} & \multirow{2}{*}{$\begin{array}{l}\text { SLASAQCPFLGPPR } \\
\text { WT }\end{array}$} \\
\hline & WT & Y87N & WT & $\mathrm{F} 102 \mathrm{C}$ & WT & F133V & & \\
\hline SPOPWT & $1.3 \pm 0.1$ & & NQ & & $2.4 \pm 0.3$ & & $6.2 \pm 0.2$ & $21.4 \pm 0.5$ \\
\hline SPOP ${ }^{Y 87 N}$ & & $61.4 \pm 2.3$ & $37.6 \pm 2.4$ & & $43.6 \pm 2.3$ & & $180 \pm 1$ & $435 \pm 9$ \\
\hline SPOPF102C & $98.6 \pm 1.0$ & & & $9.4 \pm 0.6$ & $61.1 \pm 7.7$ & & $188 \pm 2$ & $468 \pm 26$ \\
\hline SPOP F133V & $4.3 \pm 0.4$ & & NQ & & & $1.8 \pm 0.4$ & $13.9 \pm 0.03$ & $29.1 \pm 0.4$ \\
\hline
\end{tabular}

The peptide concentrations are shown in $\mathrm{fmol} / \mu \mathrm{g}$ of total protein

$N Q$ not quantified

ions to carry $4+$ charges (note that the Asp- $\mathrm{N}$ peptides also have multiple internal lysine residues and in general provide even lower MS signal). This results in small differences in $m / z$ of the precursor ions, hence the risk of having the mutant and WT peptides co-scanned (causing reduced specificity), as well as decreased fragmentation efficiency, because the beam-type collision in the triple quadrupole MS instrument is not as efficient as the electron transfer dissociation available on the hybrid Orbitrap instrument for fragmenting long, highly charged 
peptides. Moreover, these long Arg-C peptides are also highly hydrophobic, causing significant peptide loss in peptide standard handling and PRISM fractionation.

The following procedures were used to overcome these difficulties and substantially improve the assay quality: (1) high-resolution LC separation helps to fully resolve the mutant and WT peptides, eliminating potential ambiguity and interference in peptide quantification; (2) adding protein digests as a protective matrix to the pure peptide stocks effectively reduces loss of the hydrophobic Arg-C peptide internal standards; (3) use of TFA instead of the basic mobile phases in the PRISM fractionation significantly improves recovery of the SPOP peptides that have high PI values; and (4) PRISM-SRM provides higher sensitivity than regular LC-SRM for SPOP mutation analysis. As a result, all the PRISM-SRM assays showed a linear dynamic range of more than two orders of magnitude, with LOQ of $0.2-1 \mathrm{fmol} / \mu \mathrm{g}$ of total protein in the cell lysate for the mutation site(s)-containing peptides. In this report, the three most frequent SPOP mutations in prostate cancer, Y87N, F102C, and F133V, were unambiguously confirmed in respective transfected HEK293T cell lines using these PRISM-SRM assays. Since the SPOP mutations behave as loss of function mutants in cell line experiments [32], it will be important to determine in future experiments the relative abundance of mutant and wild type SPOP protein present in prostate tumor tissues.

\section{Conclusions}

In summary, PRISM-SRM provides a sensitive, isoform-specific, multiplexed, and antibody-independent approach for quantification of SPOP mutations at the protein level, which holds great promise for the verification of the mutant SPOP proteins as biomarkers for prostate cancer.

\section{Additional files}

Additional file 1: Table S1. Peptides resulted from in silico digestion using different proteolytic enzymes.

Additional file 2: Figure S1. Heat map of spectral counts of the peptides generated from recombinant SPOP protein by Arg-C and Asp-N digestion. The AAs shown in red and underlined fonts represent the reported potential mutant sites. The sequence of SPOP is obtained from UniProt (http://www.uniprot.org/).

Additional file 3: Figure S2. XICs of the transitions monitored for SPOP heavy peptides VNPKGLDEESKDYLSLYLLLVSCPKSEVR (A) and VNPKGLDEESKDYLSLCLLLVSCPKSEVR (B).

Additional file 4: Table S2. Details of the PRISM-SRM assays developed for the SPOP peptides in the mutation and non-mutation regions.

Additional file 5: Figure S3. XICS of SPOP peptide VNPKGLDEESKDYLSLYLLLVSCPKSEVR and its mutant variants VNPKGLDEESKDYLSLCLLLVSCPKSEVR and VNPKGLDEESKDYLSLNLLLVSCPKSEVR without (A) and with (B) protective matrix in the pure peptide stocks in LC-SRM analysis.
Additional file 6: Figure S4. Effect of hydrophobicity on peptide recovery (heavy/light peak area ratio of SPOP peptides without matrix versus that with matrix). SPOP peptides were ordered by their theoretical hydrophobicity values. Corresponding peptide information is listed in Additional file 4: Table S2.

Additional file 7: Figure S5. XICS of SPOP peptides in WT and HEK293T cell lines expressing WT and mutant SPOP (F133V) by regular LC-SRM analysis.

Additional file 8: Figure S6. Improvement of PRISM-SRM over LC-SRM detection of SPOP heavy peptides in the mutation region under different PRISM separation conditions: high pH (blue), 0.1\% FA (red) and 0.1\% TFA (green). The theoretical pls are marked on the top of each peptide. Corresponding peptide information is listed in Additional file 4: Table S2.

Additional file 9: Figure S7. XICs of SPOP peptides in HEK 293T cell lines expressed by SPOP-WT (A) and mutant SPOP Y87N (B), F102C (C), and F133V (D) using PRISM-SRM analysis (marked red AA means mutant substitution). Corresponding peptide information is listed in Additional file 4: Table S2.

\section{Abbreviations}

SPOP: speckle-type POZ protein; PRISM: high-pressure, high-resolution separations coupled with intelligent selection and multiplexing; SRM: selected reaction monitoring; MS: mass spectrometry; DTT: dithiothreitol; IAA: iodoacetamide; WT: wild type; HEK: human embryonic kidney; TFA: trifluoroacetic acid; CE: collision energy; LOD: limit of detection; LOQ: limit of quantification; XIC: extracted ion chromatogram.

\section{Authors' contributions}

HW, JH, YG, TS, CW, AAS, TLF, S-SC and DH carried out sample preparation, PRISM-SRM assay development, and the LC-SRM experiments. HW, TL, YG, TS, and KDR analyzed and interpreted the data. TL, KDR, CEB, JMM, MAR, DGC and JK conceived of the study and participated in its design and coordination. HW, TL, KDR, TS, W-JQ, DGC, RDS, JK, SS, CEB, JMM, and MAR contributed to the discussion and preparation of the manuscript. All authors read and approved the final manuscript.

\section{Author details}

${ }^{1}$ Biological Sciences Division, Pacific Northwest National Laboratory, P.O. Box 999, MSIN: K8-98, Richland, WA 99354, USA. ${ }^{2}$ Institute of Precision Medicine of Weill Cornell Medical College and New York Presbyterian Hospital, New York, NY, USA. ${ }^{3}$ Division of Cancer Prevention, Cancer Biomarkers Research Group, National Cancer Institute, Bethesda, MD, USA.

\section{Acknowledgements}

Portions of this work were supported by the NCI EDRN Interagency Agreement ACN15006-001 (to TL and KDR) and NIH Grant P41 RR018522 (to RDS). The experimental work described herein was performed in the Environmental Molecular Sciences Laboratory, a national scientific user facility sponsored by the Department of Energy and located at Pacific Northwest National Laboratory, which is operated by Battelle Memorial Institute for the Department of Energy under Contract DE-AC05-76RL0 1830.

\section{Competing interests}

The authors declare that they have no competing interests.

\section{Availability of data and materials}

All the Skyline-processed SRM results reported in this study can be accessed at Panorama (https://panoramaweb.org/labkey/SPOP_ProstateCancer.url).

\section{Consent for publication}

Not applicable (this study does not contain any individual person's data).

\section{Ethics approval and consent to participate}

Not applicable (this study does not involve any animal or human data or tissue). 


\section{Funding}

This work was supported by the National Cancer Institute (NCI) Early Detection Research Network (EDRN) Interagency Agreement ACN15006-001 (for study design; data collection, analysis and interpretation; and manuscript writing) and National Institutes of Health (NIH) Grant P41 RR018522 (for the initia technology development related to PRISM-SRM).

\section{Publisher's Note}

Springer Nature remains neutral with regard to jurisdictional claims in published maps and institutional affiliations.

Received: 24 May 2017 Accepted: 1 August 2017

Published online: 15 August 2017

\section{References}

1. Stratton MR, Campbell PJ, Futreal PA. The cancer genome. Nature. 2009;458(7239):719-24

2. Barbieri CE, Baca SC, Lawrence MS, Demichelis F, Blattner M, Theurillat J-P, White TA, Stojanov P, Van Allen E, Stransky N, et al. Exome sequencing identifies recurrent SPOP, FOXA1 and MED12 mutations in prostate cancer. Nat Genet. 2012;44(6):685-9.

3. Boysen G, Barbieri CE, Prandi D, Blattner M, Chae S-S, Dahija A, Nataraj S, Huang D, Marotz C, Xu L, et al. SPOP mutation leads to genomic instability in prostate cancer. Elife. 2015;4:e09207.

4. Lindberg J, Klevebring D, Liu W, Neiman M, Xu J, Wiklund P, Wiklund F, Mills IG, Egevad L, Gronberg H. Exome sequencing of prostate cancer supports the hypothesis of independent tumour origins. Eur Urol. 2013;63(2):347-53.

5. Blattner M, Lee DJ, O'Reilly C, Park K, MacDonald TY, Khani F, Turner KR, Chiu YL, Wild PJ, Dolgalev I, et al. SPOP mutations in prostate cancer across demographically diverse patient cohorts. Neoplasia. 2014;16(1):14-20

6. Abeshouse A, Ahn J, Akbani R, Ally A, Amin S, Andry CD, Annala M, Aprikian A, Armenia J, Arora A, et al. The molecular taxonomy of primary prostate cancer. Cell. 2015;163(4):1011-25.

7. Boutros PC. The path to routine use of genomic biomarkers In the cancer clinic. Genome Res. 2015;25(10):1508-13.

8. Geng C, Rajapakshe K, Shah SS, Shou J, Eedunuri VK, Foley C, Fiskus W, Rajendran M, Chew SA, Zimmermann M, et al. Androgen receptor is the key transcriptional mediator of the tumor suppressor SPOP in prostate cancer. Cancer Res. 2014;74(19):5631-43.

9. Theurillat J-PP, Udeshi ND, Errington WJ, Svinkina T, Baca SC, Pop M, Wild PJ, Blattner M, Groner AC, Rubin MA, et al. Ubiquitylome analysis identifies dysregulation of effector substrates in SPOP-mutant prostate cancer. Science. 2014;346(6205):85-9.

10. An J, Wang C, Deng Y, Yu L, Huang H. Destruction of full-length androgen receptor by wild-type SPOP, but not prostate-cancer-associated mutants. Cell Rep. 2014;6(4):657-69.

11. Geng C, He B, Xu LM, Barbieri CE, Eedunuri VK, Chew SA, Zimmermann $\mathrm{M}$, Bond R, Shou J, Li C, et al. Prostate cancer-associated mutations in speckle-type POZ protein (SPOP) regulate steroid receptor coactivator 3 protein turnover. Proc Natl Acad Sci USA. 2013;110(17):6997-7002.

12. Garcia-Flores M, Casanova-Salas I, Rubio-Briones J, Calatrava A, Dominguez-Escrig J, Rubio L, Ramirez-Backhaus M, Fernandez-Serra A, Garcia-Casado Z, Antonio Lopez-Guerrero J. Clinico-pathological significance of the molecular alterations of the SPOP gene in prostate cancer. Eur J Cancer. 2014:50(17):2994-3002.

13. Wang Q, Chaerkady R, Wu J, Hwang HJ, Papadopoulos N, Kopelovich L, Maitra A, Matthaei $\mathrm{H}$, Eshleman JR, Hruban $\mathrm{RH}$, et al. Mutant proteins as cancer-specific biomarkers. Proc Natl Acad Sci USA. 2011;108(6):2444-9.
14. Wang H, Shi T, Qian W-J, Liu T, Kagan J, Srivastava S, Smith RD, Rodland KD, Camp DG. The clinical impact of recent advances in LC-MS for cancer biomarker discovery and verification. Expert Rev Proteom. 2016;13(1):99-114.

15. Elliott MH, Smith DS, Parker CE, Borchers C. Current trends in quantitative proteomics. J Mass Spectrom. 2009;44(12):1637-60.

16. Schiess R, Wollscheid B, Aebersold R. Targeted proteomic strategy for clinical biomarker discovery. Mol Oncol. 2009;3(1):33-44.

17. Neilson KA, Ali NA, Muralidharan S, Mirzaei M, Mariani M, Assadourian G, Lee A, van Sluyter SC, Haynes PA. Less label, more free: approaches in label-free quantitative mass spectrometry. Proteomics. 2011;11(4):535-53.

18. Bantscheff M, Lemeer S, Savitski MM, Kuster B. Quantitative mass spectrometry in proteomics: critical review update from 2007 to the present. Anal Bioanal Chem. 2012;404(4):939-65.

19. Ruppen-Canas I, Lopez-Casas PP, Garcia F, Ximenez-Embun P, Munoz M, Pia Morelli M, Real FX, Serna A, Hidalgo M, Ashman K. An improved quantitative mass spectrometry analysis of tumor specific mutant proteins at high sensitivity. Proteomics. 2012;12(9):1319-27.

20. Halvey PJ, Ferrone CR, Liebler DC. GeLC-MRM quantitation of mutant KRAS oncoprotein in complex biological samples. J Proteome Res. 2012;11(7):3908-13

21. Shi T, Fillmore TL, Sun $X$, Zhao $R$, Schepmoes AA, Hossain M, Xie F, Wu S, Kim J-S, Jones N, et al. Antibody-free, targeted mass-spectrometric approach for quantification of proteins at low picogram per milliliter levels in human plasma/serum. Proc Natl Acad Sci USA. 2012;109(38):15395-400.

22. Shi T, Sun X, Gao Y, Fillmore TL, Schepmoes AA, Zhao R, He J, Moore RJ, Kagan J, Rodland KD, et al. Targeted quantification of low $\mathrm{ng} / \mathrm{mL}$ level proteins in human serum without immunoaffinity depletion. J Proteome Res. 2013;12(7):3353-61

23. Shi T, Gao Y, Quek SI, Fillmore TL, Nicora CD, Su D, Zhao R, Kagan J, Srivastava S, Rodland KD, et al. A highly sensitive targeted mass spectrometric assay for quantification of AGR2 protein in human urine and serum. $J$ Proteome Res. 2014;13(2):875-82.

24. He J, Schepmoes AA, Shi T, Wu C, Fillmore TL, Gao Y, Smith RD, Qian W-J, Rodland KD, Liu T, et al. Analytical platform evaluation for quantification of ERG in prostate cancer using protein and mRNA detection methods. J Transl Med. 2015:13:54.

25. http://www.uniprot.org

26. Kim S, Gupta N, Pevzner PA. Spectral probabilities and generating functions of tandem mass spectra: a strike against decoy databases. J Proteome Res. 2008;7(8):3354-63.

27. Kim S, Pevzner PA. MS-GF plus makes progress towards a universal database search tool for proteomics. Nat Commun. 2014;5:5277.

28. MacLean B, Tomazela DM, Shulman N, Chambers M, Finney GL, Frewen B, Kern R, Tabb DL, Liebler DC, MacCoss MJ. Skyline: an open source document editor for creating and analyzing targeted proteomics experiments. Bioinformatics. 2010;26(7):966-8.

29. Mani R-S. The emerging role of speckle-type POZ protein (SPOP) in cancer development. Drug Discov Today. 2014;19(9):1498-502.

30. Shi T, Gao Y, Gaffrey MJ, Nicora CD, Fillmore TL, Chrisler WB, Gritsenko MA, Wu C, He J, Bloodsworth KJ, et al. Sensitive targeted quantification of ERK phosphorylation dynamics and stoichiometry in human cells without affinity enrichment. Anal Chem. 2015;87(2):1103-10.

31. Anderson NL, Anderson NG, Haines LR, Hardie DB, Olafson RW, Pearson TW. Mass spectrometric quantitation of peptides and proteins using stable isotope standards and capture by anti-peptide antibodies (SISCAPA). J Proteome Res. 2004;3(2):235-44.

32. Saar M, Zhao H, Nolley R, Young SR, Coleman I, Nelson PS, Vessella RL, Peehl DM. Spheroid culture of LuCaP 147 as an authentic preclinical model of prostate cancer subtype with SPOP mutation and hypermutator phenotype. Cancer Lett. 2014:351(2):272-80. 